\title{
Quercetin Inhibits Biofilm Formation by Decreasing the Production of EPS and Altering the Composition of EPS in Staphylococcus epidermidis
}

\author{
Yongqi $\mathrm{Mu}^{1,2}$, Hong Zeng ${ }^{1,2}$ and Wei Chen 1,2,3,45* \\ ' Key Laboratory of Protection and Utilization of Biological Resources in Tarim Basin of Xinjiang Production and Construction \\ Corps, Tarim University, Alar, China, ${ }^{2}$ College of Life Sciences, Tarim University, Alar, China, ${ }^{3}$ College of Animal Sciences \\ Tarim University, Alar, China, ${ }^{4}$ Key Laboratory of Tarim Animal Husbandry and Science Technology of Xinjiang Production \\ and Construction Corps, Tarim University, Alar, China, ${ }^{5}$ Engineering Laboratory for Tarim Animal Diseases Diagnosis \\ and Control of Xinjiang Production and Construction Corps, Tarim University, Alar, China
}

OPEN ACCESS

Edited by: Muthusamy Govarthanan, University of Seoul, South Korea

Reviewed by:

Shanmugaraj Gowrishankar, Alagappa University, India

Arunachalam Kannappan, Shanghai Jiao Tong University, China

*Correspondence: Wei Chen

379497687@qq.com

Specialty section: This article was submitted to Antimicrobials, Resistance, and Chemotherapy, a section of the journal

Frontiers in Microbiology

Received: 28 November 2020

Accepted: 26 January 2021

Published: 04 March 2021

Citation:

Mu Y, Zeng H and Chen W (2021) Quercetin Inhibits Biofilm Formation by Decreasing the Production of EPS and Altering the Composition of EPS

in Staphylococcus epidermidis.

Front. Microbiol. 12:631058.

doi: 10.3389/fmicb.2021.631058
Staphylococcus epidermidis is an opportunistic pathogen, and its biofilm formation ability is an important virulent factor. Quercetin, a typical flavonoid ubiquitously used in dietary supplementation, is known for its antioxidant property, but its anti-biofilm activity against $S$. epidermidis remains unknown. In this study, the anti-biofilm activity of quercetin was investigated using S. epidermidis ATCC35984, a strong biofilm-positive strain. An attempt was made to disclose the mechanisms of the anti-biofilm activity of quercetin. S. epidermidis exhibited a less cell surface hydrophobicity after quercetin treatment. Also, quercetin effectively inhibited S. epidermidis cells from adhering to the glass slides. Quercetin downregulated the intercellular adhesion (ica) locus and then polysaccharide intercellular adhesin (PIA) production was reduced. Therefore, S. epidermidis cells became less hydrophobic, which supported quercetin's anti-biofilm effect. Our study suggests that quercetin from plants be given further attention as a potential anti-biofilm agent against the biofilm formation of $S$. epidermidis, even biofilm infections of other bacteria.

\section{Keywords: quercetin, biofilm, hydrophobicity, ica, PIA, Staphylococcus epidermidis}

\section{INTRODUCTION}

Staphylococcus epidermidis is the most frequently encountered coagulase-negative Staphylococci (CoNS) species on human skin. Characteristically, the diseases caused by S. epidermidis and other CoNS are chronic and re-occur, which contrasts the potential of $S$. aureus to cause acute infections (Lowy and Franklin, 1998). Epidemiological studies have demonstrated the presence of the genus Staphylococcus, Staphylococcus aureus, and S. epidermidis, in approximately $50 \%$ of cases of bovine mastitis (Mello et al., 2020). The consequences of mastitis include economic losses due to the costs of treatment, lower milk production, changes in product quality, and culling (Halasal et al., 2007). Besides the economic losses, mastitis is a public health hazard since it can cause zoonoses and food poisoning (Fernandes et al., 2011; Gomes et al., 2016). S. epidermidis is notorious in particular for causing infections on indwelling medical devices, including cardiac implantable electric device (CIED) infection (Okada et al., 2021) and orthopedic device-related infection (Thompson et al., 2020), in which the pathogenesis usually involves biofilm formation. Moreover, the antibiotic therapy against pathogenic bacteria is currently decreasing, which is partly attributed to biofilm 
formation (Al-Yousef et al., 2017; Singh et al., 2017). Consequently, the ability of biofilm formation by $S$. epidermidis is focused on in recent years (Vadyvaloo and Otto, 2005; Xie et al., 2019; Mu et al., 2020).

It was observed that extracts of various plants and secondary metabolites isolated from plants such as quercetin, caffeine, menthol, and chlorogenic acid have demonstrated varying levels of biofilm inhibition in gram-negative pathogens (Choo et al., 2006; Annapoorani et al., 2012; Al-Yousef et al., 2017; Satish et al., 2017; Du et al., 2018). Quercetin is a natural flavonoid antioxidant (El-Khawagah et al., 2020; Rojas et al., 2020) able to scavenge reactive species and hydroxyl radicals (Boots et al., 2008), and it bears pharmaceutical significance including anticarcinogenic (Pereira et al., 1996), anti-inflammatory (Guardia et al., 2001), and antimicrobial properties (Nitiema et al., 2012). Although the anti-biofilm/anti-virulence effects of quercetin have been explored to a wider extent in both gram-positive and gramnegative as well as a few fungi species, its anti-biofilm efficacy against Staphylococcus epidermidis has not yet been reported.

In this study, we performed scanning electron microscopy (SEM), matrix components analysis, and a hydrophobic assay to investigate the effect and mechanisms of quercetin on S. epidermidis biofilm formation.

\section{MATERIALS AND METHODS}

\section{Bacterial Strains and Growth Conditions}

S. epidermidis ATCC 35984 [intercellular adhesion (ica)positive], a strong biofilm-positive strain, was used in this study. Unless specified otherwise, tryptic soy agar/broth (TSA/TSB; Becton Dickinson, 211825) were used to culture cells at $37^{\circ} \mathrm{C}$ overnight. $\mathrm{OD}_{590}$ was measured using a spectrophotometer (BioRad) for cell growth. Each experiment was performed using at least three independent cultures.

\section{Assay for Biofilm Inhibition}

A static biofilm formation assay was performed using 96well polystyrene plates as previously described, with slight modifications (Pratt and Kolter, 1998; Xie et al., 2019; Mu et al., 2020). Briefly, cells were diluted 1:100 with fresh TSB and cultured with different concentrations of quercetin (0$1,000 \mu \mathrm{g} \mathrm{ml}^{-1}$ ) for $24 \mathrm{~h}$ without shaking at $37^{\circ} \mathrm{C}$. Biofilms were stained with crystal violet (Sigma, C3886) and dissolved in $95 \%$ ethanol $(0.5 \%, w / v)$. The optical density was measured at $490 \mathrm{~nm}$ in an enzyme-linked immunosorbent assay reader (Bio-Rad). Cell growth in the 96-well plates was also detected at $\mathrm{OD}_{590}$. Relative ability of biofilm formation was indicated as percent Relative Biofilm Formation (\%) (RBF\%), calculated by the following formula: $\mathrm{RBF} \%=$ Treated $\mathrm{OD}_{490} /$ Untreated $\mathrm{OD}_{490} \times 100 \%$. Each data point was averaged from at least 12 replicate wells (four wells from each of at least three independent cultures).

\section{Microscopic Visualization}

Biofilms grown on glass slides were stained with crystal violet and were visualized by light microscopy (Nikon Eclipse $\mathrm{Ti}$
100) at a magnification of $\times 400$ (Nithyanand et al., 2010; Xie et al., 2019; Mu et al., 2020). SEM was used to observe biofilm cells as previously described (Lee et al., 2016). Briefly, S. epidermidis strain ATCC35984 cells were diluted 1:100 with fresh TSB and inoculated onto a coverslip $\left(22 \times 22 \mathrm{~mm}^{2}\right)$ in the presence of quercetin $\left(125 \mu \mathrm{g} \mathrm{ml}^{-1}\right)$ at $37^{\circ} \mathrm{C}$ for $24 \mathrm{~h}$ without shaking.

\section{Effect of Quercetin on the Production and Components of Exopolysaccharides in S. epidermidis}

To quantify the exopolysaccharides (EPS) produced by S. epidermidis, the cells were diluted 1:100 with fresh TSB and cultured for $24 \mathrm{~h}$ with shaking at $37^{\circ} \mathrm{C}$. Then, the water extraction and alcohol precipitation method was used to collect EPS as previously described, with slight modifications (Petit and Pinilla, 1995; Li et al., 2011; Jin and Zhao, 2014), and air-dried at room temperature. The crude polysaccharides were dissolved by $0.5 \mathrm{~mol} \mathrm{~L}-1$ hydrochloric acid and $121^{\circ} \mathrm{C}$ heat treated for $15 \mathrm{~min}$. The EPS production of $S$. epidermidis was quantified using the degrees Brix assay (Ball, 2006) with or without treatment with quercetin $\left(125 \mu \mathrm{g} \mathrm{ml}^{-1}\right)$.

To detect the effect of quercetin on the components of EPS, the water extraction and alcohol precipitation method was used to collect EPS in the $S$. epidermidis culture, as above. Proteinase $\mathrm{K}$ and $n$-butyl alcohol (5:1, BOC Sciences, 71-36-3) were used to remove the proteins as described previously (Li et al., 2011). Following dialysis with distilled water overnight, the aqueous layer was collected. The liquid was lyophilized as an EPS sample for use.

Pre-column derivation high-performance liquid chromatography (HPLC) was used to detect the monosaccharide composition of EPS in S. epidermidis (Zhang et al., 2009; Xie et al., 2019; Mu et al., 2020). Ribose ( $1 \mathrm{mmol}$, per $50 \mathrm{ml})$ was used as the internal standard solution. A mixture of mannose, glucosamine, rhamnose, glucuronic acid, galacturonic acid, galactosamine, arabinose, glucose, galactose, xylose, and fucose ( $\sim 0.1 \mathrm{mmol}$ of each monosaccharide; Sigma) was dissolved in water, followed by adding $5.0 \mathrm{ml}$ of the internal standard solution. The mixture solution was then diluted to $50 \mathrm{ml}$ and retained for 1-pheny-3-methyl-5-pyrazolone (PMP; Macklin, P816062) derivation.

The chromatographic conditions were generally as follows: column, Eclipse $\mathrm{XDB}-\mathrm{C}^{18}$; temperature, $25^{\circ} \mathrm{C}$; solvent, $0.4 \%$ triethylamine in $20 \mathrm{mmol} \mathrm{L}^{-1}$ ammonium acetate buffer solution ( $\mathrm{pH} 6.3$ with acetic acid)-acetonitrile (83:17); and flow rate, $1 \mathrm{ml}$ $\mathrm{min}^{-1}$. The eluate was monitored at $245 \mathrm{~nm}$.

The correction factor for each monosaccharide $\left(f_{\mathrm{i} / \mathrm{s}}\right)$ and the content of every monosaccharide in the polysaccharide hydrolysis solution $(W)$ was calculated using the equations $f_{\mathrm{i} / \mathrm{s}}=\left(W_{\mathrm{i}} / W_{\mathrm{s}}\right) /\left(A_{\mathrm{i}} / A_{\mathrm{s}}\right)$ and $W=f_{\mathrm{i} / \mathrm{s}}\left(A_{\mathrm{i}} / A_{\mathrm{s}}\right) W_{\mathrm{s}}$, respectively. $A_{\mathrm{s}}$ and $A_{\mathrm{i}}$ are the peak areas of the internal ribose standard and the standard monosaccharide in the reference solution, respectively. $W_{\mathrm{s}}$ and $W_{\mathrm{i}}$ are the contents of the internal ribose standard and the standard monosaccharide in the reference solution, respectively. 
TABLE 1 | Primers for quantitative reverse transcriptase PCR.

\begin{tabular}{|c|c|}
\hline Genes & Primer sequences \\
\hline \multirow[t]{2}{*}{ gyrB } & 5'-TGACGAGGCATTAGCAGGTT-3' \\
\hline & 5' -GTGAAGACCGCCAGATAC $\Pi 1-3^{\prime}$ \\
\hline \multirow[t]{2}{*}{ icaR } & 5'-CATTGACGGACTITACCAGTाT-3' \\
\hline & 5'-ATCCAAAGCGATGTGCGTAG-3' \\
\hline \multirow[t]{2}{*}{$i c a B$} & 5'-GAAACAGGCTTATGGGACTाTG-3' \\
\hline & 5'-CAAGTGCGCGTTCATाாT-3' \\
\hline
\end{tabular}

\section{Cell Surface Hydrophobicity Assay}

Cell surface hydrophobicity was tested as previously described (Rosenberg et al., 1980; Xie et al., 2019; Mu et al., 2020). Briefly, $1 \mathrm{ml}$ of bacteria $\left(\mathrm{OD}_{400}=0.6\right)$ was placed into glass tubes and $250 \mu \mathrm{l}$ of $n$-hexadecane (Macklin, H810865) was added. The decrease in the $\mathrm{OD}_{400}$ of the aqueous phase was taken as a measure of $H \%$, which was calculated with the formula: $H \%=\left[\left(\mathrm{OD}_{0}-\mathrm{OD}\right) / \mathrm{OD}_{0}\right] \times 100$, where $\mathrm{OD}_{0}$ and $\mathrm{OD}$ are the $\mathrm{OD}_{400}$ before and after extraction with $n$-hexadecane, respectively. The experiments were performed using three independent cultures per condition.

\section{Quantitative Real-Time RT-PCR Assay}

To explore further the possible mechanisms of the inhibition against $S$. epidermidis biofilm by quercetin, quantitative reverse transcription PCR (qRT-PCR) was performed to investigate the transcription levels of several biofilm-associated genes in S. epidermidis ATCC35984 with and without quercetin treatment. Gene-specific primers were used for these genes and $\operatorname{gyr} B$ used as a housekeeping control (Table 1). The expression level of the housekeeping gene gyrB was used to normalize the expression data of the genes of interest. The qRT-PCR method was adapted from a previous study (Wang et al., 2011). qRT-PCR was performed using a SYBR green PCR master mix (TransGen Biotech) and an ABI PRISM 7500 Real-Time PCR System (RotorGene Q) with two independent cultures. All experiments were performed in triplicate. The $2^{-\Delta \Delta \mathrm{Ct}}$ method was used to analyze the quantitative real-time PCR data.

\section{Statistical Analysis}

GraphPad Prism 5 was used to calculate the mean and the standard deviation of the mean. All experiments were performed in triplicate and the data obtained from the experiments were presented as mean values; the difference between the control and the tested groups were analyzed using Student's $t$-test. Significant differences were $P<0.05$.

\section{RESULTS}

\section{Quercetin Inhibited Biofilm Formation by S. epidermidis in a Dose-Dependent Manner}

The results showed that quercetin inhibited the biofilm formation of S. epidermidis ATCC 35984 in a dose-dependent manner
(Figure 1A). Specifically, it decreased the biofilm formation of S. epidermidis ATCC 35984 by $\geq 90 \%$ at $250 \mu \mathrm{g} \mathrm{m}{ }^{-1}$ and by $\geq 95 \%$ at $500 \mu \mathrm{g} \mathrm{ml}^{-1}$.

The change in biofilm formation with or without quercetin treatment was observed using light microscopy and SEM. The results showed that the biofilms treated with $125 \mu \mathrm{g}$ $\mathrm{ml}^{-1}$ of quercetin became thinner, looser, and even easier to eradicate than the untreated biofilms (Figure 1B). SEM analysis revealed that fewer cells attached to the coverslips when treated with quercetin (Figure 1C). Additionally, fewer intercellular substances were present in the treated group compared with those in the untreated group (Figure 1D). No morphologic abnormality was observed in the presence of quercetin. The growth curves of $S$. epidermidis cells were also measured in the presence of quercetin $\left(125 \mu \mathrm{g} \mathrm{ml}^{-1}\right)$, and a decrease in cell growth was not observed (data not shown). The cell growth and microscopic results indicate that the inhibition by quercetin against $S$. epidermidis biofilm formation is attributed to antibiofilm activity rather than antibacterial activity.

\section{Quercetin Decreased Cell Surface Hydrophobicity}

Surface hydrophobicity facilitates adherence to hydrophobic surfaces. Thus, it plays a crucial role in biofilm formation by Staphylococci (Lee et al., 2016; Xie et al., 2019). The cell surface hydrophobicity (CSH) assay was performed to explore the mechanism underlying the inhibition by quercetin against biofilm formation by $S$. epidermidis. The results showed that $S$. epidermidis cells became less hydrophobic when treated with quercetin (Figure 2), which at least partly demonstrates the inhibitory mechanisms of quercetin on reduced biofilm formation.

\section{Quercetin Decreased the Production of EPS and Altered the Composition of EPS Produced by S. epidermidis}

In our previous work, we tested the dependent type of the biofilm formation of S. epidermidis ATCC 35984. It was found that biofilm formation by S. epidermidis ATCC 35984 mainly depends on EPS consisting of reductive polysaccharides in which the dihydroxy groups are unsubstituted (Xie et al., 2019; Mu et al., 2020). Thus, we detected the effect of quercetin on EPS. The results showed that the production of EPS by S. epidermidis ATCC 35984 was reduced when treated with quercetin (Figure 3). Specifically, for strain ATCC 35984 when treated with quercetin, galactosamine (GalN) was absent and glucose (Glu) obviously appeared in the monosaccharide composition compared with the control. Additionally, the proportion of galactose (Gal) was increased while the proportions of mannose (Man) and galacturonic acid (GalA) were decreased; especially GalA was significantly reduced (Figure 4).

\section{Quercetin Reduced PIA Production by Downregulated ica Locus}

As quercetin from plant extracts exhibited potent inhibition on the biofilm formation of S. epidermidis ATCC 35984, we 
A

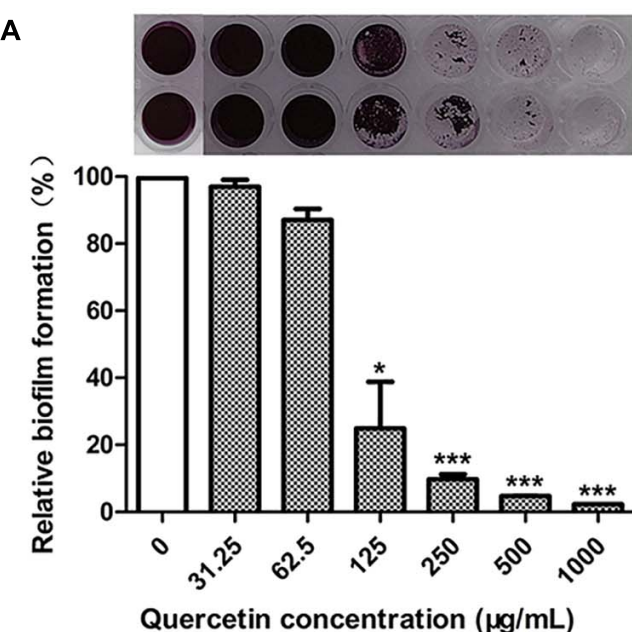

C

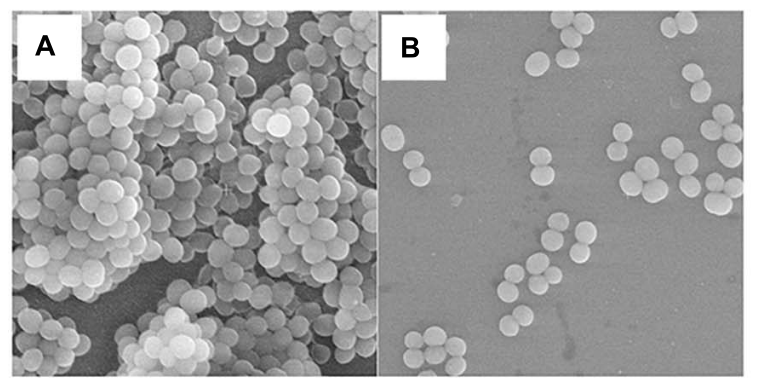

B

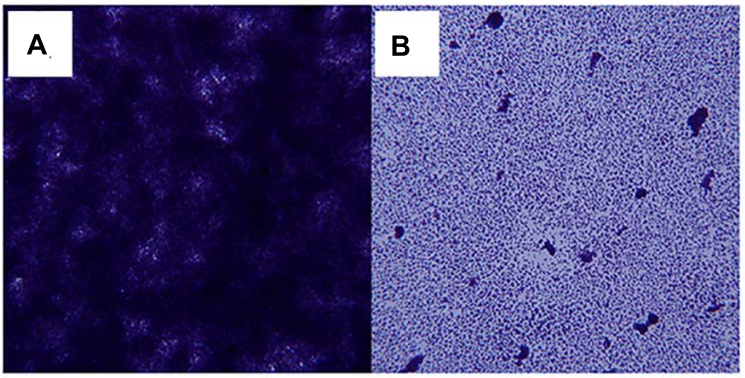

D

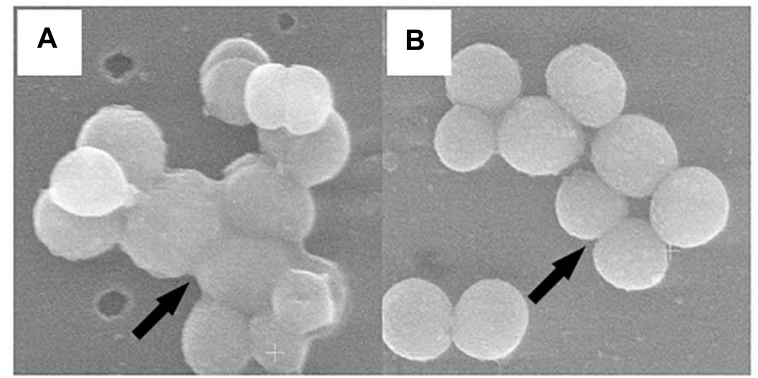

FIGURE 1 | Quercetin significantly reduced Staphylococcus epidermidis (ATCC 35984) biofilm formation. The biofilm formation (OD 490$)$ of S. epidermidis (A) was quantified at different concentrations of quercetin $\left(0-1,000 \mu \mathrm{g} \mathrm{ml}^{-1}\right)$ at $37^{\circ} \mathrm{C}$ after $24 \mathrm{~h}$ in 96 -well plates. The relative activity of biofilm formation was indicated as percent Relative Biofilm Formation (\%) (RBF\%), calculated by the following formula: RBF\% $=$ Treated $\mathrm{OD}_{490} /$ Untreated $\mathrm{OD}_{490} \times 100 \%$. Columns represent the means for three independent experiments. Error bars indicate the standard deviations. Statistically significant differences (determined by Student's $t$-test) are indicated as ${ }^{\star \star \star} P<0.001$ and ${ }^{\star} P<0.05$ vs. the control group. Biofilms formed by $S$. epidermidis ATCC 35984 were visualized by light microscopy (B) and SEM (C,D). SEM was used to examine the biofilm cells grown on coverslips in the presence of quercetin $\left(125 \mu \mathrm{g} \mathrm{ml}^{-1}\right)$. At least three independent experiments were conducted. $a$, untreated control; $b$, quercetin treated.

were curious why it could decrease polysaccharide intercellular adhesin (PIA) production. Thus, the expression of the ica locus was analyzed. Quercetin treatment resulted in a downregulation of the ica locus which was associated with the cell adhesion in S. epidermidis biofilm formation (Figure 5). The decrease of cell-to-cell adhesion caused the reduction of biofilm formation.

\section{DISCUSSION}

S. epidermidis is the main reason for biofilm-associated infections. Unlike $S$. aureus, biofilm formation by $S$. epidermidis is an important and the sole virulence factor in the onset and development of device-related infections in clinical settings. Meanwhile, the emergence of multidrug resistance among clinical pathogens has been proven to be related to biofilm formation. Thus, novel antimicrobial and antifouling agents that cannot be surpassed by those pathogens are required. Increasing evidence of plant-derived molecules as novel antimicrobials against multidrug-resistant clinical pathogens show that local special plants may become promising candidates in which novel antibiotics could be found (Packiavathy et al., 2014; Kannappan et al., 2020).

In our previous study, we found that the extract of Coreopsis tinctoria Nutt, a special local plant in Xinjiang, obviously exhibited an inhibitory effect on biofilm formation in $S$. epidermidis. Also, we tested the components in C. tinctoria Nutt and found that quercetin is rich in this local plant. Thus, we assumed that quercetin has potential anti-biofilm activity; we then chose quercetin instead of the phytochemical as our experimental subject. In the current study, quercetin was evaluated for its potential to hamper the biofilm formation in S. epidermidis. In a plate incubation assay, quercetin exhibited a concentration-dependent reduction in biofilm formation. Quercetin reduced S. epidermidis biofilm formation up to $90.5 \%$ at the concentration of $250 \mu \mathrm{g} \mathrm{ml}^{-1}$ and up to $95.3 \%$ at the concentration of $500 \mu \mathrm{g} \mathrm{ml}^{-1}$. The above results are different from those of Gopu et al. (2015), who reported 13-72, 8-80, and $10-61 \%$ reductions in the biofilm formation of 3 g-negative foodborne bacteria, Klebsiella pneumoniae, Pseudomonas aeruginosa, 


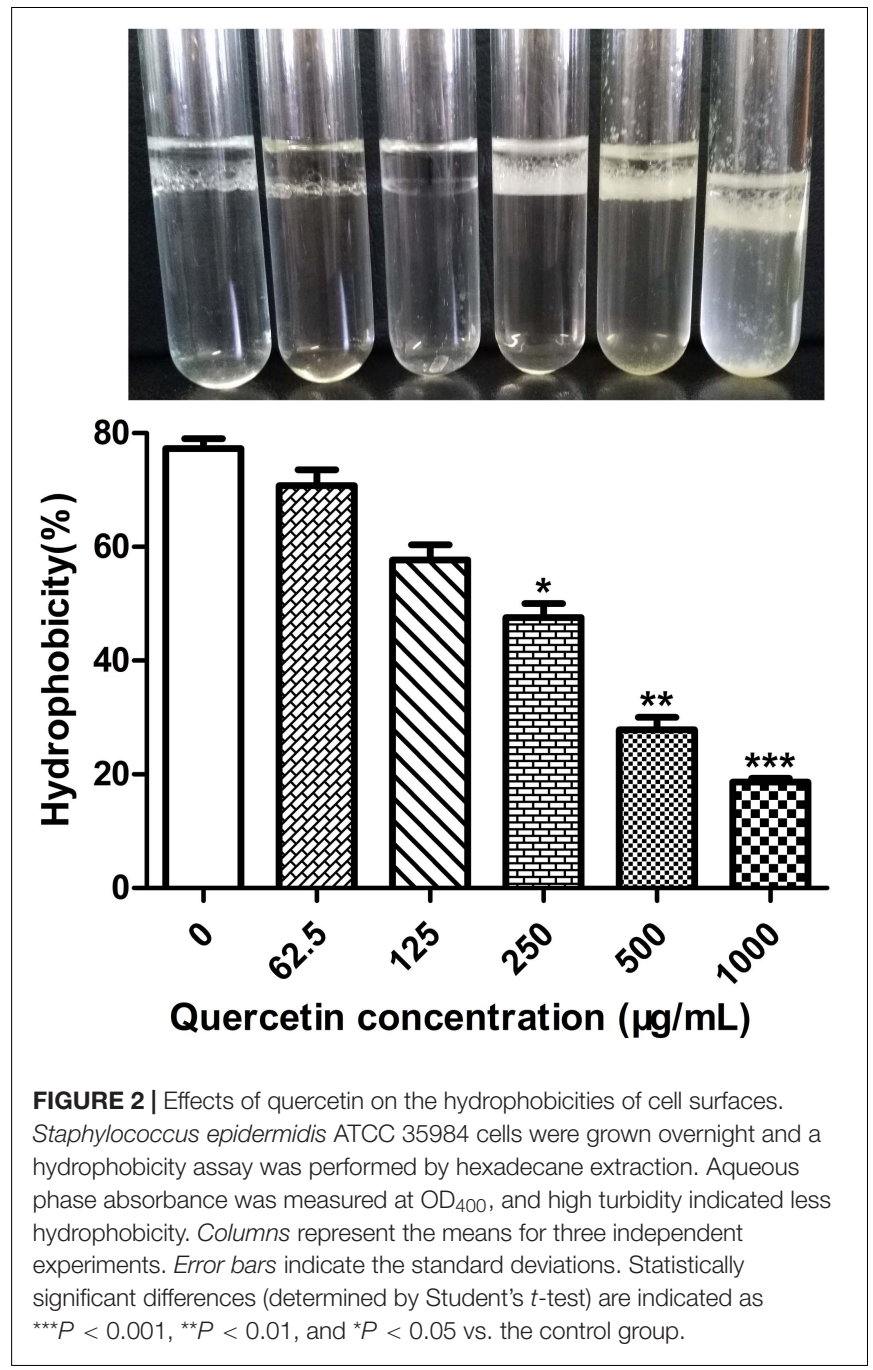

and Yersinia enterocolitica, at different concentrations of 5-40 $\mu \mathrm{g}$ $\mathrm{mL}^{-1}$, respectively.

The mechanism of $S$. epidermidis biofilm formation is a complex process in which many factors are involved. Particularly, many macromolecules, such as extracellular proteins, environmental DNA (eDNA), and EPS, are the main components in biofilms (Xie et al., 2019). Among the above three substances, the production of EPS was mainly considered as a key factor which facilitates the initial attachment of S. epidermidis. Thus, the inhibition of EPS production may cause less biofilm formation. Our results showed that quercetin had no degradation effect on proteins and DNA (see Supplementary Figures S2A,B) in this study. However, a reduced production of EPS was observed in $S$. epidermidis when treated with quercetin (Figure 3). The above result is comparable with those of Abraham et al. (2011) and Gopu et al. (2015). Also, the composition of EPS produced by $S$. epidermidis changed with treatment of quercetin (Figure 4). Our previous work also showed that the composition of $S$. epidermidis EPS was altered after being treated with spent media from Actinomycetes (Xie et al., 2019; Mu et al., 2020). However, the changed composition of EPS is different. GalN was

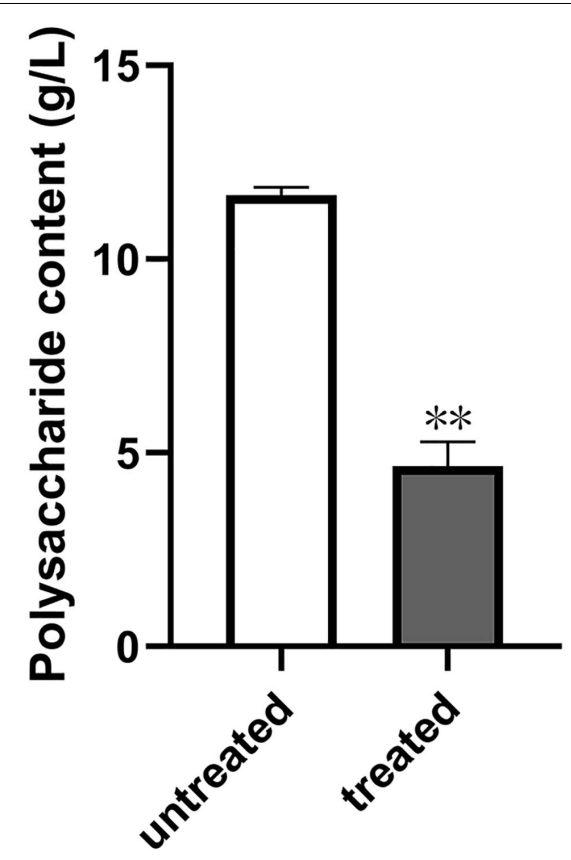

FIGURE 3 | Effect of quercetin on the exopolysaccharide (EPS) production by Staphylococcus epidermidis. Degrees Brix was used to detect the content of EPS in S. epidermidis with or without quercetin treatment $\left(125 \mu \mathrm{g} \mathrm{ml}^{-1}\right)$. Error bars indicate the standard deviations. Statistically significant differences (determined by Student's $t$-test) are indicated as ${ }^{\star \star} P<0.01$ vs. the control group.

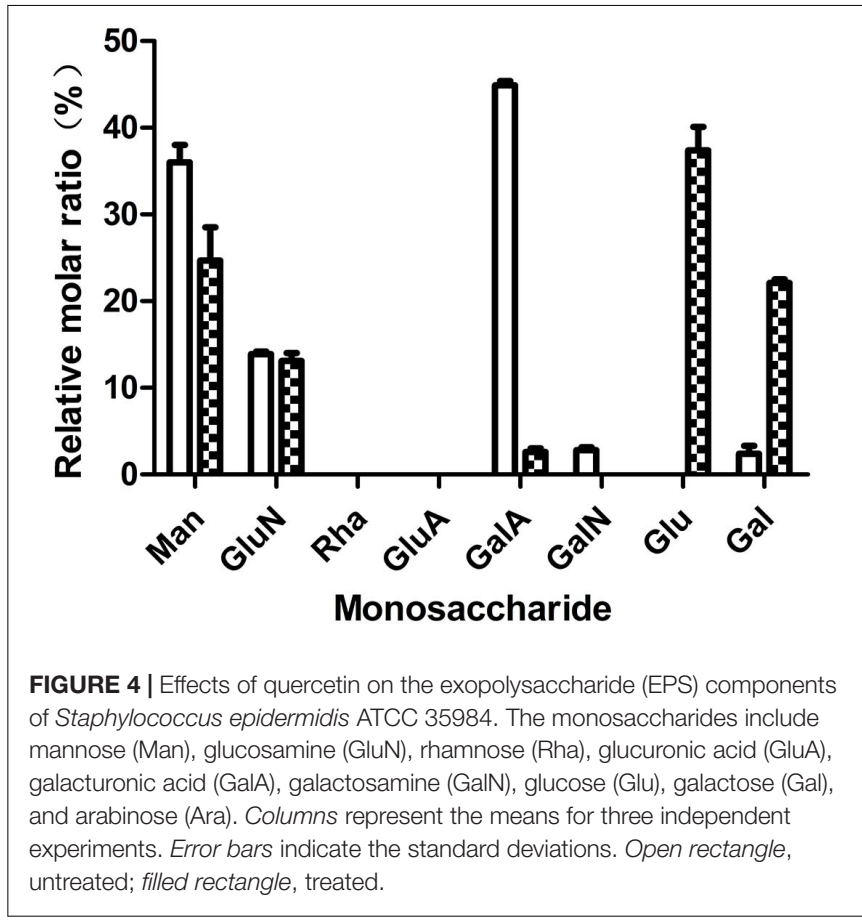

absent in quercetin treatment, while arabinose (Ara) and Gal were absent in the treatment of spent media from Actinomycetes compared with the control. GalN is a reductive glycoside that 


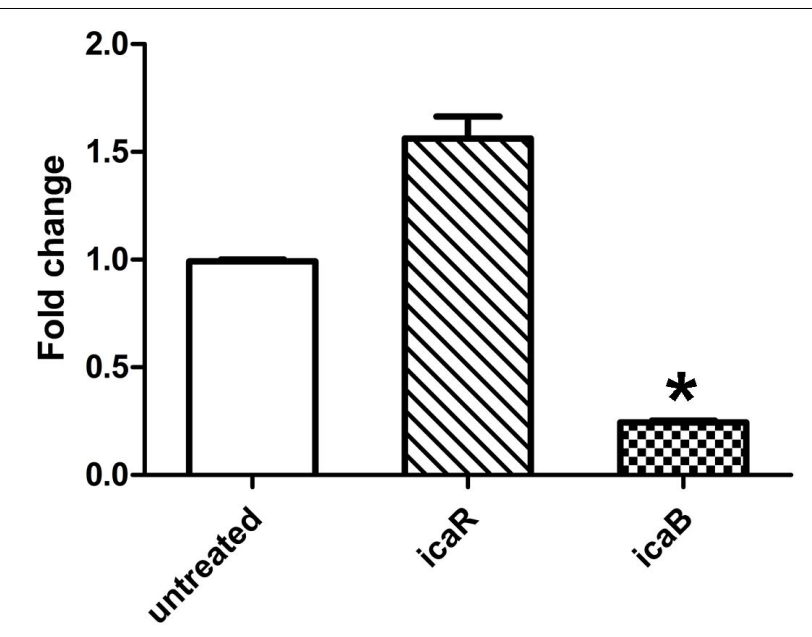

FIGURE 5 | Relative mRNA expression (mean \pm SEM) of icaB and icaR in Staphylococcus epidermidis with or without quercetin treatment $(125 \mu \mathrm{g}$ $\mathrm{ml}^{-1}$ ). Downregulation or upregulation of gene expression was considered significant when the relative expression was decreased or increased $\geq 2$-fold. All fold changes have been normalized to gyrB as the reference gene. Data are the means of fold changes with standard deviations from three independent experiments amplified in triplicate. Error bars indicate the standard deviations. Statistically significant differences (determined by Student's t-test) are indicated as ${ }^{\star} P<0.05$ vs. the control group.

plays a crucial role in S. epidermidis biofilm formation (Mack et al., 1992; Rohde et al., 2005; Xie et al., 2019). Thus, GalN disappeared when treated with quercetin, which may cause a weaker biofilm formation. Additionally, the proportions of monosaccharides of EPS were significantly different. When treated with quercetin, Gal was increased while Man and GalA were decreased. These results suggest that quercetin acts on EPS in a different manner. Further investigation will be required to understand the mechanisms.

The biofilm formation of bacteria is significantly influenced by cell surface hydrophobicity. It has been reported that hydrophobic surfaces are preferred (Xie et al., 2019; Mu et al., 2020). Enzyme-like biofilm inhibitors and low-concentration antibiotics reduce the hydrophobicity of $S$. epidermidis cells (Xie et al., 2019; Mu et al., 2020). In this study, it appears that a decreased cell surface hydrophobicity attenuates the attachment of S. epidermidis cells to the plastic wells (Figure 1A), glass slides (Figure 1B), and coverslips (Figures 1C,D). The above findings suggest that the combination of antimicrobials with cell surface hydrophobicity reducers in clinical cases increases drug sensitivity and improves the therapeutic effect.

EPS produced by $S$. epidermidis is responsible for intercellular adhesion. PIA is a type of EPS. PIA production is catalyzed by four glucuronyltransferases encoded by the icaADBC operon (Liduma et al., 2012; Mu et al., 2020), which is negatively regulated by $i c a R$, encoding a transcriptional repressor of the icaADBC operon (Conlon et al., 2002; Mu et al., 2020). The results of the relative gene expression, the downregulation of $i c a B$, and the upregulation of $i c a R$ when treated with quercetin in the current study are in agreement with this conclusion. To further explore the effect of quercetin on biofilm, we used SEM and observed that intercellular substances were reduced (Figure 1D). Meanwhile, we tested the production of $S$. epidermidis exopolysaccharides using the degrees Brix assay. In agreement with the results of SEM, a significantly decreased production of exopolysaccharides in the treated groups was found (Figure 3).

\section{CONCLUSION}

In conclusion, the results in this study indicate that quercetin exhibits anti-biofilm activity via decreasing PIA production and cell surface hydrophobicity. Quercetin warrants further attention as a potential biofilm inhibitor in biofilm-associated infections.

\section{DATA AVAILABILITY STATEMENT}

The original contributions presented in the study are included in the article/Supplementary Material, further inquiries can be directed to the corresponding author/s.

\section{AUTHOR CONTRIBUTIONS}

WC, HZ, and YM conceived and designed the experiments. YM performed the experiments. WC and YM wrote and revised the manuscript. All authors contributed to the article and approved the submitted version.

\section{FUNDING}

This work was supported by the Program for New Century Excellent Talents in University (grant no. NCET-11-1071) of China, the Program for Young and Middle-Aged Technology Innovation Leading Talents (2019CB029), and a Fund for Ph.D in Xinjiang Production and Construction Corps (2009JC07) to WC. A Microbial Resources Utilization Innovation Team in Key Field of Xinjiang Production and Construction Crops (grant no. 2017CB014) to WC.

\section{SUPPLEMENTARY MATERIAL}

The Supplementary Material for this article can be found online at: https://www.frontiersin.org/articles/10.3389/fmicb.2021. 631058/full\#supplementary-material

\footnotetext{
Supplementary Figure 1 | Effect of quercetin on initial attachment stage (A) and aggregation stage (B) in S. epidermidis biofilm formation. Biofilm formation $\left(\mathrm{OD}_{490}\right)$ of $S$. epidermidis on initial attachment stage was detected when the cells were cultured at $37^{\circ} \mathrm{C}$ for $4 \mathrm{~h}$ with the treatment of quercetin $\left(01,000 \mu \mathrm{g} \mathrm{ml}^{-1}\right)$ in 96-well plates. For determining biofilm production on aggregation stage the cells were cultured at $37^{\circ} \mathrm{C}$ for $4 \mathrm{~h}$ in 96 -well plates, followed by the treatment with different concentrations of quercetin $\left(01,000 \mu \mathrm{g} \mathrm{ml}^{-1}\right)$, and were further cultured at $37^{\circ} \mathrm{C}$ for $20 \mathrm{~h}$. Error bars indicate the standard deviations. Statistically significant differences (determined by Student's $t$-test) are indicated as ${ }^{* * *} P<0.001,{ }^{* *} P<0.01$, and ${ }^{*} P<0.05$ vs. the control group.
} 
Supplementary Figure 2 | Degradation activities of quercetin against proteins (A) and DNA (B). Protein degradation assays were performed using milk agar plates (A). Proteinase $\mathrm{K}$ and 5\% DMSO were used as positive and negative controls.

\section{REFERENCES}

Abraham, S. V. P. I., Palani, A., Ramaswamy, B. R., Shunmugiah, K. P., and Arumugam, V. R. (2011). Antiquorum sensing and antibiofilm potential of Capparis spinosa. Arch. Med. Res. 42, 658-668. doi: 10.1016/j.arcmed.2011.12. 002

Al-Yousef, H. M., Ahmed, A. F., Al-Shabib, N. A., Laeeq, S., Khan, R. A., Rehman, M. T., et al. (2017). Onion peel ethylacetate fraction and its derived constituent quercetin $4^{\prime}-\mathrm{O}-\beta-\mathrm{D}$ glucopyranoside attenuates quorum sensing regulated virulence and biofilm formation. Front. Microbiol. 8:1675-1685. doi: 10.3389/fmicb.2017.01675

Annapoorani, A., Parameswari, R., Pandian, S. K., and Ravi, A. V. (2012). Methods to determine antipathogenic potential of phenolic and flavonoid compounds against urinary pathogen Serratia marcescens. J. Microbiol. Methods. 91, 208211. doi: 10.1016/j.mimet.2012.06.007

Ball, D. W. (2006). Concentration scales for sugar solutions. J. Chem. Educ. 83, 1489-1491. doi: 10.1021/ed083p1489

Boots, A. V., Haenen, G. R., and Bast, A. (2008). Health effects of quercetin: from antioxidant to nutraceutical. Eur. J. Pharmacol. 585, 325-337. doi: 10.1016/j. ejphar.2008.03.008

Choo, J. H., Rukayadi, Y., and Hwang, J. K. (2006). Inhibition of bacterial quorum sensing by vanilla extract. Lett. Appl. Microbiol. 42, 637-641. doi: 10.1111/j. 1472-765X.2006.01928.x

Conlon, K. M., Humphreys, H., and O'Gara, J. P. (2002). icaR encodes a transcriptional repressor involved in environmental regulation of ica operon expression and biofilm formation in Staphylococcus epidermidis. J. Bacteriol. 184, 4400-4408. doi: 10.1128/jb.184.16.4400-4408. 2002

Du, D., Yao, L. B., Zhang, R., Shi, N., Shen, Y., Yang, X. M., et al. (2018). Protective effects of flavonoids from Coreopsis tinctoria Nutt. on experimental acute pancreatitis via Nrf-2/ARE-mediated antioxidant pathways. J. Ethnopharmacol. 224, 261-272. doi: 10.1016/j.jep.2018.06.003

El-Khawagah, A. R. M., Kandiel, M. M. M., and Samir, H. (2020). Effect of quercetin supplementation in extender on sperm kinematics, extracellular enzymes release, and oxidative stress of egyptian buffalo bulls frozenthawed semen. Front. Vet. Sci. 7:604460. doi: 10.3389/fvets.2020. 604460

Fernandes, J. B. C., Zanardo, L. G., Galvão, N. N., Carvalho, I. A., Nero, L. A., and Moreira, M. A. S. (2011). Escherichia coli from clinical mastitis: serotypes and virulence factors. J. Vet. Diagn. Invest. 23, 1146-1152. doi: 10.1177/ 1040638711425581

Gomes, F., Saavedra, M. J., and Henriques, M. (2016). Bovine mastitis disease/pathogenicity: evidence of the potential role of microbial biofilms. Pathog. Dis. 74:ftw006. doi: 10.1093/femspd/ftw006

Gopu, V., Meena, C. K., and Shetty, P. H. (2015). Quercetin influences quorum sensing in food borne bacteria: in-vitro and in-silico evidence. PLoS One 10:e134684. doi: 10.1371/journal.pone.0134684

Guardia, T., Rotelli, A. E., Juarez, A. O., and Pelzer, L. E. (2001). Anti-inflammatory properties of plant flavonoids. Effects of rutin, quercetin and hesperidin on adjuvant arthritis in rat. Farmaco 56, 683-687. doi: 10.1016/S0014-827X(01) 01111-9

Halasal, T., Huijpsl, K., Osterds, O., and Hogeveen, H. (2007). Economic effects of bovine mastitis and mastitis management: a review. Vet. Q. 29, 18-31. doi: 10.1080/01652176.2007.9695224

Jin, X., and Zhao, S. (2014). Extraction optimization and bioactivities of an extracellular polysaccharide produced by Aspergillus fumigatus. Int. J. Biol. Macromol. 68, 13-17. doi: 10.1016/j.ijbiomac.2014.04.024

Kannappan, A., Durgadevi, R., Srinivasan, R., Lagoa, R. J. L., Packiavathy, I. A. S. V., Pandian, S. K., et al. (2020). Hemidesmus indicus2-Hydroxy-4methoxybenzaldehyde from is antagonistic to biofilm formation. Biofouling 36, 549-563. doi: 10.1080/08927014.2020.1777989

Lee, J. H., Kim, Y. G., Lee, K., Kim, C. J., and Lee, J. (2016). Streptomycesderived actinomycin D inhibits biofilm formation by Staphylococcus aureus
Protein digestion was observed by a clear zone surrounding proteinase $\mathrm{K}$. DNA degradation of Staphylococcus epidermidis by quercetin was tested at $37^{\circ} \mathrm{C}$ for $2 \mathrm{~h}$ (B). DNase I was used as a positive control.

and its hemolytic activity. Biofouling 32, 45-56. doi: 10.1080/08927014.2015.11 25888

Li, X., Zhao, R., Zhou, H. L., and Wu, D. H. (2011). Deproteinization of polysaccharide from the stigma maydis by sevag method. Adv. Mater. 340, 416-420. doi: 10.4028/www.scientific.net/amr.340.416

Liduma, I., Tracevska, T., Bers, U., and Zilevica, A. (2012). Phenotypic and genetic analysis of biofilm formation by Staphylococcus epidermidis. Medicina (Kaunas) 48, 305-309.

Lowy, F. D., and Franklin, D. (1998). Staphylococcus aureus infections. New Engl. J. Med. 339, 520-532.

Mack, D., Siemssen, N., and Laufs, R. (1992). Parallel induction by glucose of adherence and a polysaccharide antigen specific for plastic-adherent Staphylococcus epidermidis: evidence for functional relation to intercellular adhesion. Infect. Immun. 60, 2048-2057. doi: 10.1128/iai.60.5.2048-2057. 1992

Mello, P. L., Riboli, D. F. M., Martins, L. A., Brito, M. A. V. P., Victória, C., Calixto Romero, L., et al. (2020). Staphylococcus spp. Isolated from bovine subclinical mastitis in different regions of Brazil: molecular typing and biofilm gene expression analysis by RT-qPCR. Antibiotics (Basel) 9:888. doi: 10.3390/ antibiotics9120888

Mu, Y. Q., Xie, T. T., Zeng, H., Chen, W., Wan, C. X., and Zhang, L. L. (2020). Streptomyces-derived actinomycin D inhibits biofilm formation via downregulating ica locus and decreasing production of PIA in Staphylococcus epidermidis. J. Appl. Microbiol. 128, 1201-1207. doi: 10.1111/jam. 14543

Nithyanand, P., Thenmozhi, R., Rathna, J., and Pandian, S. K. (2010). Inhibition of Streptococcus pyogenes biofilm formation by coral-associated actinomycetes. Curr. Microbiol. 60, 454-460. doi: 10.1007/s00284-009-9564-y

Nitiema, L. W., Savadogo, A., Simpore, J., Dianou, D., and Traore, A. S. (2012). In vitro antimicrobial activity of some phenolic compounds (coumarin and quercetin) against gastroenteritis bacterial strains. Int. J. Microbiol. Res. 3, 183-187. doi: 10.5829/idosi.ijmr.2012.3.3.6414

Okada, A., Tabata, H., Shoda, M., Shoin, W., Kobayashi, H., Okano, T., et al. (2021). Safe and effective transvenous lead extraction for elderly patients utilizing nonlaser and laser tools: a single-center experience in Japan. Heart Vessels doi: 10.1007/s00380-020-01761-3 [Epub ahead of print]

Packiavathy, I. A. S. V., Priya, S., Pandian, S. K., and Ravi, A. V. (2014). Inhibition of biofilm development of uropathogens by curcumin - an anti-quorum sensing agent from Curcuma longa. Food Chem. 148, 453-460. doi: 10.1016/j.foodchem. 2012.08.002

Pereira, M. A., Grubbs, C. J., Barnes, L. H., Li, H., Olson, G. R., Eto, I., et al. (1996). Effects of the phytochemicals, curcumin and quercetin, upon azoxymethane-induced colon cancer and 7, 12-dimethylbenz [a] anthraceneinduced mammary cancer in rats. Carcinogenesis 17, 1305-1311. doi: 10.1093/ carcin/17.6.1305

Petit, M. D., and Pinilla, J. M. (1995). Production and purification of a sugar syrup from carob pods. LWT - Food Sci. Technol. 28, 145-152. doi: 10.1016/s00236438(95)80027-1

Pratt, L. A., and Kolter, R. (1998). Genetic analysis of Escherichia coli biofilm formation: roles of flagella, motility, chemotaxis and type I pili. Mol. Microbiol. 30, 285-293. doi: 10.1046/j.1365-2958.1998.01061.x

Rohde, H., Burdelski, C., Bartscht, K., Hussain, M., Buck, F., Horstkotte, M. A., et al. (2005). Induction of Staphylococcus epidermidis biofilm formation via proteolytic processing of the accumulation-associated protein by staphylococcal and host proteases. Mol. Microbiol. 55, 1883-1895. doi: 10.1111/j.1365-2958. 2005.04515.x

Rojas, L. S., Torres, G. S., Quiles, C. L., Gomez-Caturla, J., Garcia-Garcia, D., and Balart, R. (2020). On the use of phenolic compounds present in citrus fruits and grapes as natural antioxidants for thermo-compressed bio-based highdensity polyethylene films. Antioxidants (Basel) 10:14. doi: 10.3390/antiox10 010014

Rosenberg, M., Gutnick, D., and Rosenberg, E. (1980). Adherence of bacteria to hydrocarbons: a simple method for measuring cell-surface hydrophobicity. 
FEMS. Microbiol. Lett. 9, 29-33. doi: 10.1111/j.1574-6968.1980.tb05 599.x

Satish, K. R., Samiraj, R., Ann, S. S., and Lee, J. (2017). Antibiofilm and anti$\beta$-lactamase activities of burdock root extract and chlorogenic acid against Klebsiella pneumoniae. J. Microbiol. Biotechnol. 27, 542-551. doi: 10.4014/jmb. 1609.09043

Singh, V. K., Mishra, A., and Jha, B. (2017). Anti-quorum sensing and anti-biofilm activity of delftia tsuruhatensis extract by attenuating the quorum sensingcontrolled virulence factor production in Pseudomonas aeruginosa. Front. Cell Infect. Microbiol. 7:337. doi: 10.3389/fcimb.2017.00337

Thompson, K., Freitag, L., Styger, U., Camenisch, K., Zeiter, S., Arens, D., et al. (2020). Impact of low bone mass and antiresorptive therapy on antibiotic efficacy in a rat model of orthopedic device-related infection. J. Orthop. Res. 39, 415-425. doi: 10.1002/jor.24951

Vadyvaloo, V., and Otto, M. (2005). Molecular genetics of Staphylococcus epidermidis biofilms on indwelling medical devices. Int. J. Artif. Organs 28, 1069-1078. doi: 10.1177/039139880502801104

Wang, X., Niu, C., Sun, G., Dong, D., Villaruz, A. E., and Li, M. (2011). Ygs is a novel gene that influences biofilm formation and the general stress response of Staphylococcus epidermidis. Infec. Immun. 79, 1007-1015. doi: 10.1128/IAI. 00916-10

Xie, T. T., Zeng, H., Ren, X. P., Wang, N., Chen, Z. J., Zhang, Y., et al. (2019). Antibiofilm activity of three Actinomycete strains against Staphylococcus epidermidis. Lett. Appl. Microbiol. 68, 73-80. doi: 10.1111/lam.13087

Zhang, Z. S., Shi, X. L., Wang, J., Zhang, Q. B., and Zhang, J. J. (2009). Analysis of the monosaccharide composition of fucoidan by precolumn derivation HPLC. Chin. J. Oceanol. Limnol. 27, 578-582. doi: 10.1007/s00343-009-9205-0

Conflict of Interest: The authors declare that the research was conducted in the absence of any commercial or financial relationships that could be construed as a potential conflict of interest.

Copyright (C) $2021 \mathrm{Mu}$, Zeng and Chen. This is an open-access article distributed under the terms of the Creative Commons Attribution License (CC BY). The use, distribution or reproduction in other forums is permitted, provided the original author(s) and the copyright owner(s) are credited and that the original publication in this journal is cited, in accordance with accepted academic practice. No use, distribution or reproduction is permitted which does not comply with these terms. 